\title{
Infecciones asociadas a la atención en salud (IAAS) en pacientes pediátricos post-operados de cardiopatías congénitas
}

\author{
José Barriga, Jaime Cerda, Katia Abarca, Marcela Ferrés, Paula Fajuri, \\ María Riquelme, Diego Carrillo y Cristián Clavería
}

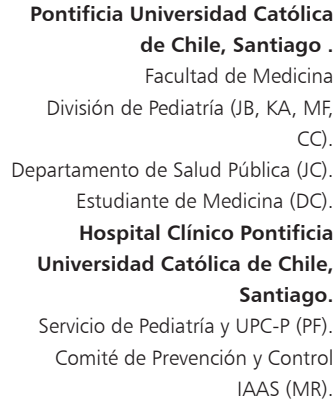

Pontificia Universidad Católica de Chile, Santiago Facultad de Medicina División de Pediatría (JB, KA, MF,

Departamento de Salud Pública (JC)

Estudiante de Medicina (DC)

Hospital Clínico Pontificia Universidad Católica de Chile, Santiago. Servicio de Pediatría y UPC-P (PF)

Comité de Prevención y Contro IAAS (MR)

Declaración de conflictos de interés: Ninguno Fuente de financiamiento: Ninguno.

Recibido: 28 de marzo de 2013 Aceptado: 18 de diciembre de

Correspondencia a: Cristián Clavería Rodríguez claveria@med.puc.c

\section{Introducción}

L as infecciones asociadas a la atención en salud (IAAS) son responsables de una elevada morbilidad y mortalidad en pacientes pediátricos sometidos a cirugías cardíacas. Se han reportado incidencias que van de 16 a 31\%, más alta que la observada en los pacientes hospitalizados en unidades de cuidados intensivos pediátricos general. Está ampliamente reportada su asociación con aumento en los días de hospitalización, costos asociados a la intervención, uso de antimicrobianos y riesgo de mortalidad $^{1-8}$.

Los tipos más comunes de IAAS reportadas en pacientes pediátricos post operados de cirugía cardíaca son las infecciones del sitio quirúrgico y del torrente sanguíneo. Entre los factores de riesgo que han sido identificados previamente están: menor edad al momento de la cirugía, mayor complejidad quirúrgica, mayor duración de la cirugía y del bypass cardiopulmonar, mayor estadía en la unidad de cuidados intensivos y retraso en el cierre esternal $^{1-8}$

El Hospital Clínico de la Pontificia Universidad Católica de Chile (PUC) es un centro de referencia nacional para la resolución de las cardiopatías congénitas operables en menores de 15 años. Desde el año 2005 fue incorporado como un centro prestador del Ministerio de Salud dentro del programa de Garantías Explícitas en Salud (GES) para la atención y resolución de cardiopatías congénitas9. Se ha observado un aumento progresivo y sostenido en el número de procedimientos cardio-quirúrgicos realizados por año en nuestro centro. Durante el 2010 se realizaron 211 cirugías, con una mortalidad operatoria de $4,7 \%{ }^{10}$.

El objetivo del estudio fue determinar los factores de riesgo que inciden en la ocurrencia de IAAS en los pacientes postoperados de cardiopatías congénitas admitidos en la Unidad de Paciente Crítico Pediátrico (UPC-P) del Hospital Clínico de la PUC.

\section{Pacientes y Métodos}

Estudio de casos y controles que incluyó pacientes menores de 15 años portadores de cardiopatía congénita que fueron sometidos a cirugía cardíaca (reparadora o paliativa) desde enero de 2007 a diciembre de 2011, admitidos en la UPC-P del Hospital Clínico de la PUC. Se definió como casos, aquellos pacientes que presentaron IAAS, dentro de las cuales fueron consideradas: infección 
del sitio quirúrgico, infección del tracto urinario asociada al uso de catéter urinario permanente, neumonía asociada a ventilación mecánica e infección del torrente sanguíneo asociada al uso de catéter venoso central, diagnosticadas de acuerdo a las normas nacionales ${ }^{11,12}$; y que fueron identificadas y registradas por el Comité de Prevención y Control de IAAS del hospital. Para este estudio se analizó la información del primer episodio de infección, ya que en este grupo de pacientes podría darse la ocurrencia de más de una infección durante la misma hospitalización.

Se consideró como controles, aquellos pacientes operados inmediatamente después de un caso y que no presentaron IAAS, para lo cual se utilizó el registro de las cirugías de cardiopatías congénitas realizadas en nuestra Institución.

Se realizó una revisión retrospectiva de la base de datos de cirugías cardíacas congénitas, así como una revisión de fichas clínicas y registros de enfermería de la UPC-P. Se clasificaron los posibles factores de riesgo para el desarrollo de IAAS en aquellos identificables al ingreso del paciente a la UPC-P y aquellos que se desarrollan durante la evolución del paciente.

Se consideraron como factores de riesgo identificables al ingreso del paciente a la UPC-P: edad, sexo, peso al ingreso, presencia de síndrome genético, fisiología de corazón univentricular, tipo de cardiopatía congénita, categorización de la complejidad de la cirugía según score RACHS-1 del inglés "Risk Adjustment for Surgery for Congenital Heart Disease (ajuste del riesgo para cirugía de cardiopatía congénita) ${ }^{13-14}$ y el tiempo de circulación extracorpórea (CEC) durante la cirugía.

Entre los factores de riesgo que podrían desarrollarse durante la estadía del paciente en UPC-P se consideraron: apoyo inotrópico-vasoactivo durante las primeras $24 \mathrm{~h}$ de evolución (SIV: score inotrópico-vasoactivo) ${ }^{15}$; días de uso de catéter venoso central, línea auricular, catéter urinario permanente, ventilación mecánica; necesidad y días con tórax abierto; uso de hemoderivados, nutrición parenteral, corticosteroides sistémicos; días de mantención de la profilaxis antimicrobiana; y tiempo de estadía en UPC-P.

El estudio fue aprobado por el Comité de Ética de la Dirección de Investigación y del Comité de Prevención y Control de IAAS del Hospital Clínico de la PUC.

\section{Análisis estadístico}

Las variables categóricas fueron descritas en términos de número y porcentaje, y las variables numéricas en términos de mediana y rango. La asociación entre variables categóricas fue evaluada mediante el Test Exacto de Fisher, y la asociación entre variables categóricas y numéricas mediante el Test de Mann-Whitney. Para identificar aquellas variables asociadas a IAAS se construyó un modelo de regresión logística (modalidad: Enter) incluyendo las variables identificables al ingreso del paciente a UPC-P que hubiesen presentado un valor $\mathrm{p}<0,05$ en el análisis univariado y que no estuviesen correlacionadas.

\section{Resultados}

Se incluyeron 39 pacientes con un primer episodio de IAAS. La mediana de edad fue dos meses, 23 (59\%) eran de género masculino. La mediana de peso fue $4,4 \mathrm{~kg}$ (r: 2,6-91). Un síndrome genético estuvo presente en 8 pacientes $(20,5 \%)$, principalmente síndrome de Down (6 pacientes). Cardiopatías con una fisiología univentricular estuvieron presentes en 15 pacientes (38,5\%). Las principales cardiopatías congénitas fueron: hipoplasia de ventrículo izquierdo en $6(30,8 \%)$, transposición de grandes arterias en $5(12,8 \%)$, tetralogía de Fallot en 5 $(12,8 \%)$, comunicación interventricular en $4(10,2 \%)$ y canal aurículo-ventricular en 4 (10,2\%). Veintiocho $(71,8 \%)$ de los pacientes tuvieron un procedimiento de mediana a mayor complejidad según la categorización RACHS-1. Se produjo mortalidad operatoria en 3 pacientes $(7,3 \%)$ (Tabla 1$)$.

La sospecha de infección se estableció en una mediana de 6 días de evolución de post-operatorio. La infección del sitio quirúrgico fue la infección más frecuente, presente en 21 pacientes $(53,8 \%)$, seguida por la infección urinaria en 11 pacientes $(28,2 \%)$. La infección del torrente sanguíneo asociada al uso de catéter venoso central estuvo presente en $5(12,8 \%)$ y la neumonía asociada a ventilación mecánica en $2(5,1 \%)$.

Dentro del grupo de pacientes con infección de sitio quirúrgico 13 pacientes tuvieron una infección superficial, 2 pacientes una infección profunda y 6 pacientes desarrollaron mediastinitis. Los microorganismos causantes identificados se describen en la Tabla 2.

\begin{tabular}{|lcc|}
\hline \multicolumn{4}{|c|}{ Tabla 1. Características de pacientes con IAAS post cirugía cardíaca } \\
\hline Masculino & $\mathbf{n}=39$ & $\%$ \\
Edad, mediana en meses (rango) & 23 & 59 \\
Peso, mediana en kgs (rango) & $2(0-175)$ & 38,5 \\
Cardiopatía con fisiología univentricular & $4,4(2,6-91)$ & \\
Cardiopatías congénitas: & 15 & 30,8 \\
Hipoplasia de ventrículo izquierdo & & 12,8 \\
Transposición de grandes arterias & 12 & 12,8 \\
Tetralogía de Fallot & 5 & 10,2 \\
Comunicación interventricular & 5 & 10,2 \\
Canal auriculo-ventricular & 4 & 7,3 \\
Estadía en UPC-P, mediana en días, (rango) & 4 & $(3-64)$ \\
Mortalidad operatoria & 3 & \\
\hline
\end{tabular}


Tabla 2. Tipos de infecciones y microorganismos aislados post cirugía cardíaca ( $\mathrm{n}$ : 39)

\begin{tabular}{|c|c|c|}
\hline Tipo de IAAS & n $\quad(\%)$ & Microorganismos \\
\hline ISQ & $21(53,8)$ & $\begin{array}{l}\text { Gram+: S. aureus SM (5), SCN (5) } \\
\text { Gram-: P. aeruginosa (1) } \\
\text { Hongos: C. albicans (1), Aspergillus (1) } \\
\text { Cultivo negativo: } 8\end{array}$ \\
\hline ITU/CUP & $11(28,2)$ & $\begin{array}{l}\text { Gram+: E. faecalis (1) } \\
\text { Gram-: E. coli (3), E. cloacae (2), P. aeruginosa (2), } \\
\text { K. pneumoniae (2), E. aerogenes (1), K. oxytoca (1) } \\
\text { Hongos: C. tropicalis (1) }\end{array}$ \\
\hline ITS/CVC & $5(12,8)$ & $\begin{array}{l}\text { Gram+: S. epidermidis (2), SCN (1) } \\
\text { Gram-: K. pneumoniae BLEE (1), E. asburiae (1) }\end{array}$ \\
\hline NAVM & $2(5,1)$ & Gram-: P. aeruginosa (1), E. cloacae (1) \\
\hline \multicolumn{3}{|c|}{$\begin{array}{l}\text { ISQ: Infección de sitio quirúrgico; ITU/CUP: Infección urinaria asociada a catéter urinario permanente } \\
\text { ITS/CVC: Infección del torrente sanguíneo asociada a catéter venoso central; NAVM: neumonía aso- } \\
\text { ciada a ventilación mecánica. SM: sensible a meticilina. SCN: Staphylococcus coagulasa negativa. SM } \\
\text { sensible a meticilina. }\end{array}$} \\
\hline
\end{tabular}

Tabla 3. Análisis univariado de factores de riesgo para infecciones post cardiocirugía presentes al ingreso a la UPC-P

\begin{tabular}{lccc} 
& Con IAAS n: $\mathbf{3 9}$ & Sin IAAS $\mathbf{n : 3 9}$ & $\mathbf{p}$ \\
Sexo masculino (\%) & $23(59)$ & $22(56,4)$ & 1,000 \\
Edad, mediana en meses (rango) & $2(0-175)$ & $5(0-108)$ & 0,003 \\
Peso, mediana en kg (rango) & $4,4(2,67-91)$ & $5,8(3-44)$ & 0,041 \\
Sindrome genético (\%) & $8(20,5)$ & $11(28,2)$ & 0,599 \\
Univentricular (\%) & $15(38,5)$ & $6(15,5)$ & 0,040 \\
Complejidad según RACHS-1 (\%) & & & 0,003 \\
1 & $2(5,1 \%)$ & $6(15,4 \%)$ & \\
2 & $9(23,1 \%)$ & $20(51,3 \%)$ & \\
3 & $14(35,9 \%)$ & $11(28,2 \%)$ & \\
4 & $3(7,7 \%)$ & $1(2,6 \%)$ & \\
6 & $11(28,2 \%)$ & $1(2,6 \%)$ & \\
Tiempo CEC** $\geq 200$ min (\%) & $17(43,6 \%)$ & $2(5,1 \%)$ & $<0,001$ \\
\hline *RACHS-1: riesgo ajustado en cirugía de cardiopatía congénita. **CEC: Circulación extracorpórea. &
\end{tabular}

Tabla 4. Análisis univariado de factores de riesgo para infecciones post cardiocirugía que se desarrollan durante la hospitalización en UPC-P

\begin{tabular}{|c|c|c|c|}
\hline & $\begin{array}{c}\text { Con IAAS } \\
n=39\end{array}$ & $\begin{array}{c}\text { Sin IAAS } \\
n=39\end{array}$ & $\mathbf{P}$ \\
\hline SIV primeras $24 \mathrm{~h}$, mediana(r) & $14(0-38)$ & $7(0-30)$ & $<0,001$ \\
\hline Días de CVC, mediana ( $r$ ) & $7(0-54)$ & $3(0-9)$ & $<0,001$ \\
\hline Días de línea auricular, mediana (r) & $6(0-36)$ & $0(0-7)$ & $<0,001$ \\
\hline Días CUP, mediana ( $r$ ) & $7(0-32)$ & $2(0-8)$ & $<0,001$ \\
\hline Días VM, mediana ( $r$ ) & $6(0-62)$ & $1(0-9)$ & $<0,001$ \\
\hline Días con profilaxis antimicrobiana, mediana $(r)$ & $3(1-14)$ & $2(1-7)$ & 0,025 \\
\hline Cierre esternal diferido (\%) & $17(43)$ & $2(5)$ & $<0,001$ \\
\hline Uso de hemoderivados (\%) & $25(64,1)$ & $8(20,5)$ & $<0,001$ \\
\hline Uso de nutrición parenteral (\%) & $18(46,2)$ & $5(12,8)$ & 0,002 \\
\hline Uso de corticosteroides sistémicos (\%) & $12(30,8)$ & $3(7,7)$ & 0,019 \\
\hline Días de estadía en UPC-P, mediana ( $r$ ) & $10(r: 3-64)$ & $4(r: 1-11)$ & $<0,001$ \\
\hline
\end{tabular}

SIV: Score inotrópico-vasoactivo; CVC: catéter venoso central; CUP: catéter urinario permanente; VM: ventilación mecánica. $r=$ rango.
De los factores de riesgo identificables al ingreso del paciente a UPC-P, mediante análisis univariado, se observó diferencias estadísticamente significativas entre pacientes infectados y no infectados en cuanto a edad, peso, presencia de una fisiología univentricular, complejidad según RACHS-1 y tiempo de CEC $\geq 200$ min. No se observó diferencias en cuanto al sexo y a la presencia de síndrome genético (Tabla 3 ).

En el análisis univariado de factores de riesgo que se presentan durante la evolución del paciente en UPC-P, se observó diferencias estadísticamente significativas en todas las variables estudiadas (Tabla 4).

Por último, se realizó un análisis multivariado incluyendo sólo aquellos factores que tendrían la capacidad de predecir la ocurrencia de IAAS (peso, fisiología univentricular, RACHS-1 y CEC $\geq 200$ min); no se incluyó la variable edad por estar altamente correlacionada con la variable peso. El análisis demostró que sólo el tiempo de CEC $\geq 200$ minutos OR 11,57 (IC: 1,04-128,5) sería el principal factor de riesgo para el desarrollo de IAAS en esta serie de pacientes. (Coeficiente $\mathrm{R}^{2}$ : 0,35. Test Hosmer y Lemeshow: $p=0,216)$.

\section{Discusión}

El estudio realizado permite conocer las IAAS que se presentan en pacientes pediátricos post-operados de cardiopatías congénitas en un centro de referencia nacional. Infecciones que deben ser consideradas por su capacidad de complicar y prolongar el proceso de recuperación post-operatoria. Se identificó como principal causa de IAAS a la infección del sitio quirúrgico, ocasionadas principalmente por bacterias grampositivas.

Al momento de realizar el análisis univariado de los factores de riesgo para el desarrollo de IAAS, se consideró que éstos deberían ser separados en aquellos que están presentes desde que se inicia el manejo post-operatorio y que podrían ser identificados al ingreso del paciente a la UPC-P; y aquellos factores que se van desarrollando durante la evolución del paciente en la UPC-P. De esta forma se observó que la edad, el peso, la presencia de una cardiopatía con fisiología univentricular, mayor complejidad del procedimiento y el tiempo de CEC $\geq 200$ min serían factores de riesgo a considerar al momento que el paciente ingresa a la unidad de paciente crítico. Se estableció un tiempo de CEC $\geq 200$ minutos por tratarse de un factor de riesgo de mayor mortalidad quirúrgica previamente identificado $^{10}$. El sexo y la presencia de un síndrome genético no fueron factores de riesgo para el desarrollo de infecciones en esta revisión.

Entre los factores estudiados que se desarrollan durante la evolución post-operatoria se observó que los pacientes que desarrollaron IAAS tuvieron más días de 
uso de catéter venoso central, línea auricular, catéter urinario permanente y ventilación mecánica. Este grupo de pacientes necesitó mayor soporte inotrópico-vasoactivo durante las primeras 24 horas de evolución; necesitaron más hemoderivados, recibieron corticosteroides sistémicos y nutrición parenteral. La necesidad de cierre esternal diferido fue mayor en estos pacientes y con una estadía más prolongada en la unidad de paciente crítico.

Los factores de riesgo estudiados concuerdan con los descritos en otros estudios ${ }^{16-24}$. Levy y cols. ${ }^{16}$, describieron como factores de riesgo: la edad neonatal, mayor complejidad del procedimiento, estadía prolongada en UPC-P y tórax abierto postoperatorio. La estadía prolongada en UPC-P ha sido asociada con el desarrollo de infección ${ }^{18}$, pero este factor no estaría claro si sería causa o consecuencia de la infección.

La edad del paciente menor a 6 meses, estadía mayor a 48 h en UPC-P y esternón abierto por más de 48 horas fueron considerados por Alegra y cols., como factores predictores para un mayor riesgo de infección ${ }^{1}$. La estadía prolongada está comúnmente asociada con mayor duración de la ventilación mecánica y aumento en el tiempo de uso de catéteres intravasculares ${ }^{25-28}$. El retardo en el cierre esternal está asociado con mayor riesgo de infecciones del sitio quirúrgico y con la ocurrencia de infecciones del torrente sanguíneo ${ }^{29}$. Estos antecedentes han permitido, recientemente, el desarrollo de modelos de predicción que tiene como objeto ayudar al médico clínico en la toma de decisiones y evaluación de las medidas de prevención implementadas en los pacientes de mayor riesgo, dentro de los cuales destacan la evaluación de las medidas antimicrobianas y decisión en la antibioprofilaxis de rutina ${ }^{1,30}$.

Clavería y cols. ${ }^{10}$, describieron la edad menor a 1 mes y el tiempo de CEC $\geq 200$ min como factores de riesgo para mayor mortalidad quirúrgica (los resultados de este estudio se encuentran en proceso de publicación). Para el presente trabajo, el análisis de regresión logística demostró que el tiempo de CEC $\geq 200$ min fue el principal factor de riesgo asociado con la ocurrencia de IAAS.

Está descrito cómo la cirugía cardíaca y el uso de CEC conlleva una activación de mecanismos inflamatorios. La etiología es multifactorial, por contacto de la sangre con los componentes del circuito, la injuria por isquemiareperfusión y el trauma quirúrgico. Esto representa una causa importante de deterioro de la función inmune, ya que ocasiona disminución del número de linfocitos y deterioro de la función de neutrófilos. Este compromiso inmunológico se correlaciona con la ocurrencia y desarrollo de secuelas clínicas post-operatorias como una respuesta inflamatoria sistémica, sepsis y disfunción de órganos ${ }^{31-33}$.

Los resultados sugieren que los pacientes postoperados de cardiopatías congénitas requieren de un manejo multidisciplinario altamente especializado, que incluya la prevención de IAAS mediante la evaluación constante de las medidas antimicrobianas empleadas, antibioprofilaxis de rutina utilizada, conducta activa de "desinvadir" precozmente y el uso juicioso de la terapia medicamentosa (antimicrobianos, hemoderivados, corticosteroides sistémicos, etc.).

\section{Resumen}

Introducción: Las infecciones asociadas a la atención en salud (IAAS) generan elevada morbilidad y mortalidad en niños sometidos a cirugía cardíaca. Objetivo: Determinar factores de riesgo que inciden en la ocurrencia de IAAS en niños operados de cardiopatías congénitas. Métodos: Estudio retrospectivo de casos y controles, en pacientes menores de 15 años sometidos a cirugía por cardiopatías congénitas desde enero 2007 a diciembre 2011 admitidos en la Unidad de Paciente Crítico Pediátrico (UPC-P) de un hospital universitario. Para los casos, se analizó la información del primer episodio de infección. Resultados: Se enrolaron 39 pacientes con IAAS y 39 controles operados que no desarrollaron infección. La mediana de edad de los casos fue 2 meses. Se identificó una serie de factores asociados a la ocurrencia de IAAS, destacando en el análisis univariado: edad, peso, fisiología cardíaca tipo univentricular, complejidad del procedimiento quirúrgico según RACHS-1 y tiempo de circulación extracorpórea $(\mathrm{CEC}) \geq 200 \mathrm{~min}$. El análisis multivariado identificó al tiempo de CEC $\geq 200 \mathrm{~min}$ como el principal factor de riesgo, con un OR de 11,57 (IC: 1,04-128,5). Conclusión: El tiempo de CEC $\geq 200$ min fue el factor de riesgo más asociado al desarrollo de IAAS en niños operados de cardiopatías congénitas.

\section{Referencias bibliográficas}

1.- Algra S, Driessen M, Schadenberg A, Schouten A, Haas F, Bollen C, et al. Bedside prediction rule for infections after pediatric cardiac surgery. Intensive Care Med 2012; 38: 474-81.

2.- $\quad$ Abou R, Najm H, Balky H, Bullard L, Kabbani M. Impact of bloodstream infection on the outcome of children undergoing cardiac surgery. Pediatr Cardiol 2010; 31: 483-9.

3.- Grisaru-Soen G, Paret G, Yahav D, Boyko V, Lerner-Geva L. Nosocomial infections in pediatric cardiovascular surgery patients: a 4-year survey. Pediatr Crit Care Med 2009; 10: 202-6.

4.- Sarviki E, Lyytikainen O, Nieminen H, Sairanen H, Saxen H. Nosocomial infections after pediatric cardiac surgery. Am J Infect Control 2008; 36: 564-9.

5.- Guardia M, Jordan I, Urrea M. Infección nosocomial en postoperados de cirugía cardíaca. An Pediatr (Barc) 2008; 69(1): 34-8.

6.- Kagen J, Lautenbach E, Bilker W, Matro J, Bell L, Domínguez T, et al. Risk factors for mediastinitis following median sternotomy in children. Pediatr Infect Dis J 2007; 26: 613-8. 
7.- Nateghian A, Taylor G, Robinson J. Risk factors for surgical site infections following open-heart surgery in a Canadian pediatric population. Am J Infect Control 2004; 32: 397-401.

8.- Rosanova M, Allaria A, Santillan A, Hernández C, Landry L, Ceminara R, et al. Risk factors for infection after cardiovascular surgery in children in Argentina. Braz J Infect Dis 2009; 13 (6): 414-6.

9.- Ministerio de Salud de Chile. Guía clínica. Cardiopatías congénitas operables en menores de 15 años. www.minsal.cl/portal/url/item/720bf efe91e0d2ede04001011f010ff2.pdf (accedido el 3 de septiembre de 2012).

10.- Clavería C, Cerda J, Schiele C, Barreno B, Becker P, Urcelay G, et al. Mortalidad operatoria y estratificación de riesgo en pacientes pediátricos operados de cardiopatía congénita: experiencia de 10 años. En proceso de publicación. Rev Chilena Cardiología (enviado).

11.- Fica A, Cifuentes M, Hervé B. Actualización del Consenso "Neumonía asociada a ventilación mecánica" Primera parte. Aspectos diagnósticos. Rev Chilena Infectol 2011; 28 (2): 130-51.

12.- García P, Payá E, Olivares R, Cotera A, Rodríguez J, Sanz M. Consenso. Diagnóstico de las infecciones asociadas a catéteres vasculares centrales. Rev Chilena Infectol 2003; 20 (1): 41-50.

13.- Jenkins K, Gauvreau K. Center-specific differences in mortality: preliminary analyses using the risk adjustment in congenital heart surgery (RACHS-1) method. J Thorac Cardiovas Surg 2002; 124: 97-104.

14.- Jenkins K, Gauvreau K, Newburger J, Spray T, Moller J, Iezzoni L. Consensus-based method for risk adjustment for surgery for congenital heart disease. J Thorac Cardiovasc Surg 2002; 123: $110-8$.

15.- Gaies M, Gurney J, Yen A, Napoli M, Gajarski R, Ohye R, et al. Vasoactive-inotropic score as a predictor of morbidity and mortality in infants after cardiopulmonary bypass. Pediatr
Crit Care Med 2010; 11: 234-8.

16.- Levy I, Ovadia B, Erez E, Rinat S, Ashkenazi S, Birk S, et al. Nosocomial infections after cardiac surgery in infants and children: incidence and risk factors. J Hosp Infection 2003; 53: 111-6.

17.- Sohn A, Schwartz J, Yang K, Jarvis W, Guglielmo J, Weintrub P. Risk factors and risk adjustment for surgical site infections in paediatric cardiothoracic surgery patients. Am J Infect Control 2010; 38; 706-10.

18.- Mehta P, Cunningham C, Colella C, Alferis G, Weiner L. Risk factors for sternal wound and other infections in pediatric cardiac surgery patients. Pediatr Infect Dis J 2000; 19: 1000-4.

19.- Bezzio S, Scolfaro C, Broglia R, Calabrese R, Mignone F, Abruzzesse P, et al. Prospective incidence study of bloodstream infection in infants and children with central venous catheters after cardiac surgery in Italy. Infect Control Hosp Epidemiol 2009; 30: 698-701.

20.- Marelli A, Gauvreau K, Landzberg M, Jenkins K. Sex differences in mortality in children urdergoing congenital heart disease surgery. A United States population-based study. Circulation 2010; 122: S234-S240.

21.- Pasquali S, Halla M, Li J, Peterson E, Jaggers J, Lodge A, et al. Corticosteroids and outcome in children undergoing congenital heart surgery. Circulation 2010; 122: 2123-30.

22.- Sarvikivi E, Lyytikäinen O, Nieminen H, Sairanen H, Saxén H. Nosocomial infections after pediatric cardiac surgery. Am J Infect Control 2008; 36: 564-9.

23.- Shah S, Kagen J, Lautenbach E, Bilker W, Matro J, Dominguez T, et al. Bloodstream infections after median sternotomy at a children`s hospital. J Thorac Cardiovasc Surg 2007; 133: 345-40

24.- Yogaraj J, Elward A, Fraser V. Rate, risk factors and outcomes of nosocomial primary bloodstream infection in pediatric intensive care unit patiens. Pediatrics 2002; 110: 481-5.

25.- Valera M, Scolfaro C, Capello N, Gramaglia E, Grassitelli S, Abbate MT, et al. Nosocomial infections in pediatric cardiac surgery, Italy. Infect Control Hosp Epidemiol 2001; 22: 771-5.

26.- Urrea M, Pons M, Serra M, Latorre C, Palomeque A. Prospective incidence study of nosocomial infections in a pediatric intensive care unit. Pediatr Infect Dis J 2003; 22: 490-4.

27.- Allpress AL, Rosenthal GL, Goodrich KM, Lupinetti FM, Zerr DM. Risk factors for surgical site infections after pediatric cardiovascular surgery. Pediatr Infect Dis J 2004; 23: 231-4.

28.- Costello JM, Graham DA, Morrow DF, Potter-Bynoe G, Sandora TJ, Laussen PC. Risk factors for central line associated bloodstream infection in a pediatric cardiac intensive care unit. Pediatr Crit Care 2009; 10: 543-9.

29.- Das S, Rubio A, Simsic JM, Kirshbom PM, Kogon B, Kanter KR, et al. Bloodstream infections increased after delayed sterna closure: cause or coincidence. Ann Thorac Surg 2011; 91: 793-7.

30.- Barker GM, O'Brien SM, Welke KF, Jacobs JP, Benjamin DK Jr, Peterson ED, et al. Major infection after pediatric cardiac surgery: a risk estimation model. Ann Thorac Surg 2010; 89: 843-850.

31.- Gessler P, Pfenninger J, Pfammatter JP, Carrel T, Baenziger O, Dahinden C. Plasma levels of interleukin-8 and expression of interleukin-8 receptors on circulating neutrophils and monocytes after cardiopulmonary bypass in children. J Thorac Cardiovasc Surg 2003; 126: 718-25.

32.- Allan C, Newburger J, McGrath E, Elder J, Psoinos C, Laussen $\mathrm{P}$, et al. The relationship between inflammatory activation and clinical outcome after infant cardiopulmonary bypass. Anesth Analg 2010; 111: 1244-51.

33.- Shi S, Shi C, Zhao Z, Shen H, Fang X, Tan L, et al. Effect of open heart surgery with cardiopulmonary bypass on peripheral blood lympochyte apoptosis in children. Pediatr Cardiol 2009; 30: 153-9. 